\title{
Erratum to: Clinical role of HER2 gene amplification and chromosome 17: a study on 154 IHC-equivocal cases of invasive breast carcinoma patients
}

\author{
Muhammad Afzal ${ }^{1,2} \cdot$ Mohammed Amir ${ }^{2} \cdot$ Muhammad Jawad Hassan $^{3}$. \\ Muhammad Sikander Hussain ${ }^{1} \cdot$ Muhammad Naveed Aziz $^{1} \cdot$ Sheeba Murad $^{3}$. \\ Iram Murtaza ${ }^{1}$. Mariam Anees ${ }^{1} \cdot$ Aneesa Sultan ${ }^{1}$
}

Published online: 30 September 2016

(C) International Society of Oncology and BioMarkers (ISOBM) 2016

Erratum to: Tumor Biol. (2016) 37:8665-8672

DOI 10.1007/s13277-015-4657-7

The original version of this article unfortunately contained an error in Materials and methods section under Patients/ specimen selection.

The corrected text under first paragraph now reads as follow:

\section{Materials and methods \\ Patients/specimen selection}

A cohort of 154 IHC-equivocal (2+) cases of female invasive breast carcinoma patients were recruited between 2012 and February 2014 from sources including referral clinical labs, oncology clinics and Surgical Department of Shifa International Hospital (SIH), Pakistan (including both in hospitalized and referral cases). The paraffin-embedded tissues and surgical specimens immunohistochemically diagnosed as weak HER2 oncoprotein overexpression were included in the study and retrospectively analyzed by dual-probe FISH assay for the clinical assessment of HER2 oncogene and aneusomy 17 in the Cancer Genetics Lab of Quaid-iAzam University, Islamabad, Pakistan by collaborating with Molecular Biologists and Cytogeneticians. The study protocol was approved by the Institutional Review Board and Ethical Committee (IRB-EC) of SIH and Shifa College of Medicine (SCM) with a reference number (IRB-EC \#2069-118-2013).

The online version of the original article can be found at http://dx.doi. org/10.1007/s13277-015-4657-7.

Aneesa Sultan

aneesasultan@gmail.com; aneesa@qau.edu.pk

1 Department of Biochemistry, Quaid-i-Azam University, Islamabad 45320, Pakistan

2 Shifa International Hospital, H-8/4, Islamabad, Pakistan

3 Atta-ur-Rehman School of Applied BioSciences (ASAB), National University of Science and Technology (NUST), Islamabad, Pakistan 